\title{
The study of the local director orientation in chiral liquid crystals
}

\author{
E. S. Kraynyukov, A. A. Karetnikov, A. P. Kovshik, \\ E. I. Ryumtsev, E. V. Aksyonova, V. P. Romanov \\ The Faculty of Physics of the Saint Petersburg State University, \\ 198504, St. Petersburg, Petrodvorets, Russia \\ bacoom888@gmail.com, akaret@mail.ru,sashakovshik@yandex.ru, \\ rei39@mail.ru, aksev@mail.ru, vpromanov@mail.ru
}

\section{PACS 42.70.Df; 42.25.Bs; 42.25.Fx; 42.25.Gy DOI 10.17586/2220-8054-2015-6-4-470-478}

\begin{abstract}
The refraction of an extraordinary light wave in a layer of a chiral liquid crystal with the director rotated $90^{\circ}$ has been studied. In this structure, if a light wave is incident on the surface of the liquid crystal at a large angle and the light passes through the whole layer, it refracts at the opposite boundary of the layer. The angular dependence of the light transmission through the liquid crystal layer has been researched. It has been demonstrated that the electric field changed the character of the refraction of the extraordinary wave and the light started to pass through the layer. The threshold voltage of the light transmission has been determined, as well as its dependence on the light incidence angle. The effect dynamics have been studied and the angular dependence of the light transmission decay time has been determined.
\end{abstract}

Keywords: refraction, chiral liquid crystal, relaxation time.

Received: 20 June 2015

\section{Introduction}

The use of liquid crystals (LC) in display technology is based on the control of the optical properties of LC thin layers with the help of an electric field. Under the influence of an electric field, the director of an LC (the direction of preferential orientation of molecules) changes its position in space, affecting the intensity of the light transmitting through the liquid crystal layer. Associated with the director reorientation, the light transmission switch-on and switch-off times $\tau_{\text {on }}$ and $\tau_{\text {off }}$ are the most important performance characteristics of liquid crystal devices. Due to this, there is a need to study the changes of orientation structure of liquid crystals under the influence of external electric fields, as well as the dependence of $\tau_{\text {on }}$ and $\tau_{\text {off }}$ on various parameters for liquid crystal layers. The research of the LC director reorientation dynamics at different distances from the LC layer boundary is of particular interest.

Such investigation can be performed for a liquid crystal layer with a helical structure, using the phenomena of refraction [1-3]. Refraction was observed in plain liquid crystal layers, displaced between glass surfaces, on the surface of which the director was parallel to the surfaces and had the same direction. In the middle of the layer, the director was rotated $90^{\circ}$ from the director position at the boundaries. The extraordinary ray, incident from the glass to the liquid crystal layer at angles exceeding the angle of total internal reflection of the ordinary ray, entered the LC, refracted inside the layer, and returned back through the same glass surface. Calculated predictions showed that the distance from the surface layer to the ray refraction point decreased, when the angle of incidence for the light to the LC layer was increased. The application of an electric field led to distortion of the LC layer structure orientation and a change in the ray's path. When the magnitude of the electric field exceeded the Fréedericksz threshold, 
refraction was disturbed - the ray passed through the layer. In works [3-5], the intensity of the transmitted light, the threshold voltage, and the cell transmission switch-on and switch-off time dependencies on the light angle of incidence were studied. Varying the angle of incidence allowed us to study the change in the local orientation of the LC director under the influence of an electric field with a smooth change of the depth of the ray penetration into the layer.

When using liquid crystals in a variety of electro-optical devices, there are several sets of boundary conditions that allow the creation of the necessary structure for LC director orientation, thereby changing the optical characteristics of the devices. Therefore, comparison of the characteristics for a system with $180^{\circ}$ rotation of the director, studied in works [3-5], with the characteristics of other helical structures having different boundary conditions is of great scientific and practical interest. In this regard, the purpose of this work was to study light refraction in the layer of the plane-oriented, chiral liquid crystal (used in works [3-5]), in which the direction of the director on surfaces differs by $90^{\circ}$.

\section{Experiment}

The experimental cell for studying the refraction in chiral liquid crystal layers was composed of two glass trapezoidal prisms (1) and (2) with a base size of $50 \times 25 \mathrm{~mm}$ and height of $18 \mathrm{~mm}$ (Fig. 1). The inclination of the entrance faces to the base was $68^{\circ}$. The refractive index of the prisms $n_{\mathrm{gl}}$ was 1.7002 for the wavelength $\lambda=632.8 \mathrm{~nm}$. There were transparent conductive coatings (electrodes) and thin polyimide layers at the prism bases. By rubbing the latter, the planar orientation was set with the surface for the liquid crystal providing strong anchoring for the LC director. At the same time, the LC director at the layer's first boundary, where the ray of light was incident, was directed perpendicular to the plane of the figure (Fig. 1). At the second boundary of the layer the director lies in the plane of the figure. The required LC layer thickness of $8 \mu \mathrm{m}$ was set with the help of Teflon spacers. The space between the prisms was filled with the chiral liquid crystal mixture (previously used in works $[4,5]$ ) with the helical pitch $P_{0}=32 \mu \mathrm{m}$ that consisted of LC-1466 (NIOPIK) with the chiral dopant VIH-3 (Vilnius State University, Lithuania). The dielectric anisotropy $\Delta \varepsilon$ of LC-1466 was 11.7 for a frequency of $1 \mathrm{kHz}$. The principal refractive indices values for the ordinary and extraordinary rays $n$ and $n_{0}$, for wavelength $\lambda=632.8 \mathrm{~nm}$ were 1.691 and 1.511 respectively. For a given layer thickness and helical pitch, the LC director was rotated by $90^{\circ}$ when passing from one layer's boundary to the other.

The scheme of the experimental setup is shown in Fig. 2 (top view). The beam of light from a helium-neon laser with wavelength $\lambda=632.8 \mathrm{~nm}$ and the diameter of $1 \mathrm{~mm}$ was incident to the studied cell with the liquid crystal (LC-cell) through the half-wave plate $\lambda / 2$. With the half-wave plate, the polarization vector of the incident ray was parallel to the LC director at the interface between the liquid crystal and glass. Next, the light fell on the photodetector $\mathrm{Ph}$, whose signal was recorded with the digital oscilloscope Osc (ASK-3106) and the computer. For signal control, we used a bipolar rectangular voltage generator ANR-3122, forming voltage pulses up to $10 \mathrm{~V}$ with the duration of $0.1-5.0$ seconds and carrier frequency $f=1 \mathrm{kHz}$. The controlled voltage from the generator was applied to the electrodes of the cell and the oscilloscope. In order to change the angle of incidence $\delta$ for the ray to the liquid crystal layer, the cell was mounted on the rotating table with the angle-reading device with an accuracy of 1 minute. 


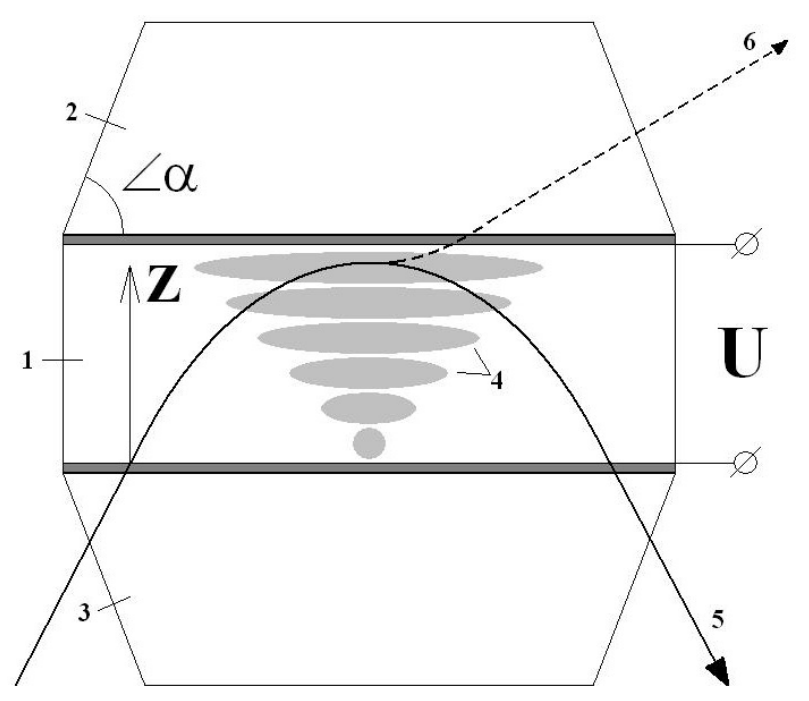

FIG. 1. LC-cell: 1 - liquid crystal layer; 2,3 - glass prisms; 4 - director configuration in liquid crystal layer; 5 - the trajectory of the extraordinary ray in the absence of an electric field; 6 - the trajectory of the extraordinary ray when electric field is applied; $U$ - control voltage

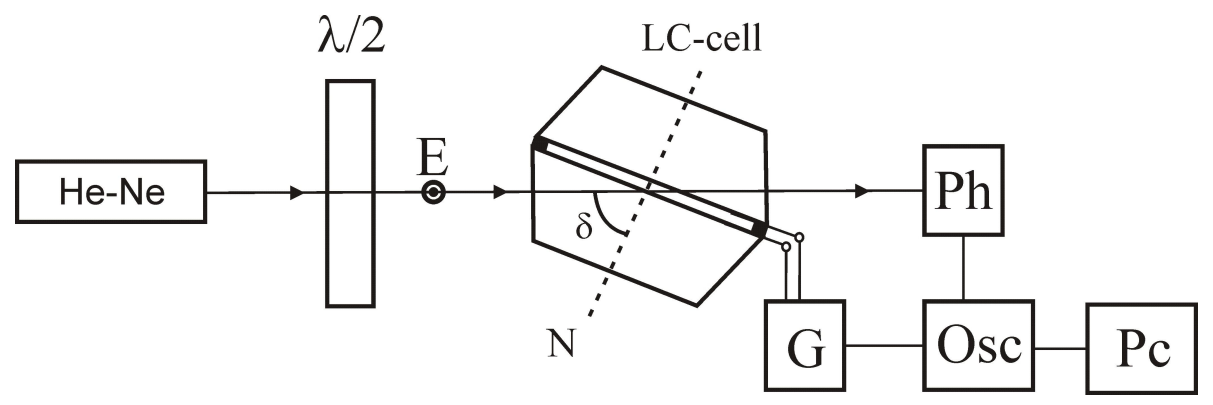

FIG. 2. Scheme of experimental setup: He-Ne - laser; $\lambda / 2$ - half wave plate; LCcell - liquid crystal cell; $\mathrm{Ph}$ - photodetector; $\mathrm{G}$ - generator; Osc - oscilloscope; $\mathrm{PC}$ - computer

\section{Experimental results}

Primarily, the dependence of the intensity of the light passing through the cell at the angle of incidence for the extraordinary ray to the LC layer was obtained (Fig. 3). The figure shows that with angles of incidence smaller than the critical angle of total internal reflection for the ordinary ray $\left(\delta=62.7\right.$ when $\left.n_{0}=n_{\perp}=1.511\right)$, the intensity of the transmitted light is maximized. The observed intensity oscillations in the areas of max transmission of the cell may be caused by interference between the rays refracted from the first and second layer boundaries, since a change in the angle of incidence results in a phase difference change between the interfering rays. In the $62.0^{\circ}-63.1^{\circ}$ angular range, the LC-cell light transmission changes from its maximum value to zero. The decreasing intensity deviations from monotony in this area are also associated with the interference of the rays reflected from both boundaries of the LC layer. The value of the critical angle of refraction (the extraordinary ray return back near second interface), which was $62.7^{\circ}$ (within experimental error), coincided with the limit angle of refraction for the LC-cell which had its director rotated by $180^{\circ}$ [3-5]. 


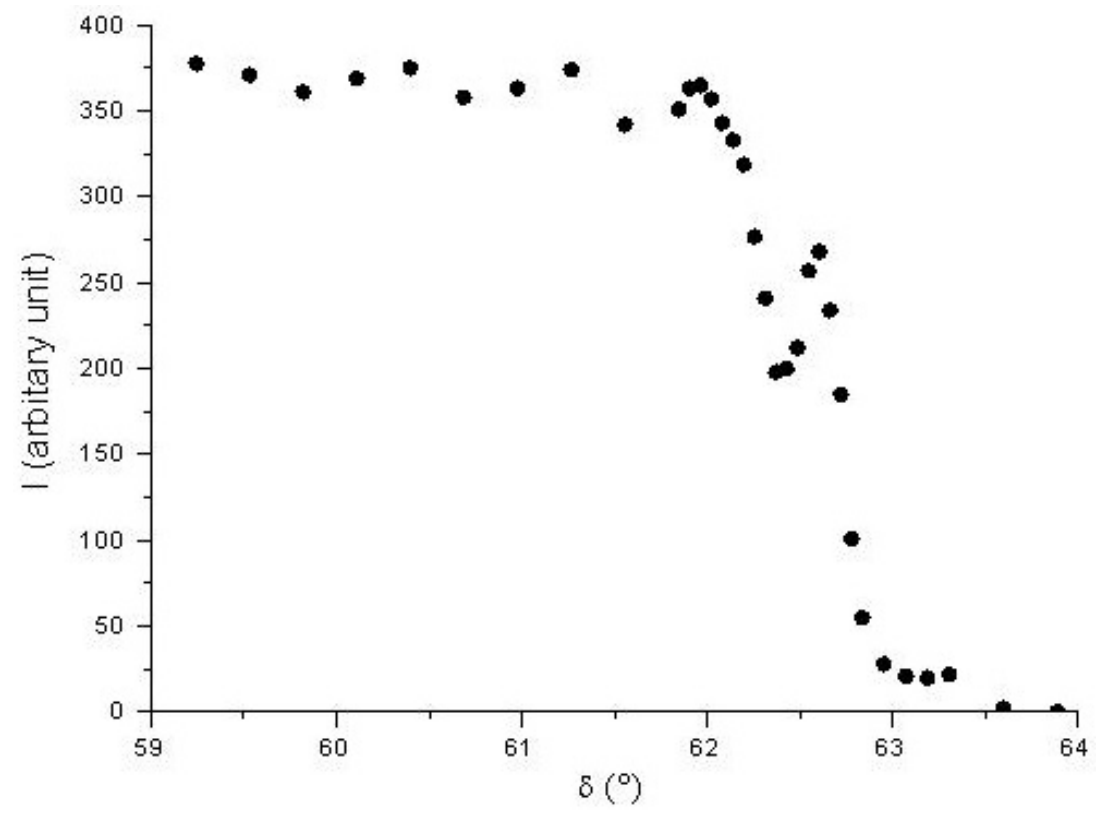

FIG. 3. The dependence of the intensity $I$ of the light passing through the cell on the incidence angle $\delta$ of the extraordinary ray to the LC layer

With angles of incidence larger than the critical angle of refraction $\left(62.7^{\circ}\right)$, the effects of the electric field of sufficient magnitude on the cell led to the disturbance (cut-off) of extraordinary ray refraction and light passing through the cell. The oscillograms of the control voltage $U=8 \mathrm{~V}$ at frequency of $1 \mathrm{kHz}$ (a) and the optical response (b) for an angle of incidence of $63^{\circ}$ are shown in Fig. 4. For comparison, there are also optical responses of the LC-cell to the applied electric field, when the light falls perpendicular to the LC layer in an electricallycontrolled birefringent mode (c) and the cut-off of the Mauguin regime (d), specific to the twist cells with a $90^{\circ}$ rotation of the director. From the obtained oscillograms, it follows that the times for establishing maximum transmission in these modes differ. The time for complete refraction cut-off $\tau_{1}$ is significantly higher than the similar parameter in electrically-controlled birefringent modes $\tau_{2}$ and the cut-off of the Mauguin regime $\tau_{3}$. Upon cancellation of the electric field, the fastest cessation for light transmission is specific to the refraction recovery mode.

The refraction cut-off mode, similar to the electrically-controlled birefringent modes and the Mauguin regime cut-off, has a threshold value. The threshold voltage of light transmission through the cell $U_{\text {th }}$ was determined by using the dependence of the transmitted light intensity $I$ on the applied voltage $U$. As an example, Fig. 5 shows the dependence of $I$ on $U$ for the angle of incidence of $62.8^{\circ}$.

The increase in the light angle of incidence to the liquid crystal layer within the $62.8^{\circ}-$ $79.7^{\circ}$ range resulted in an increase in the threshold voltage from $1.1 \mathrm{~V}$ to $8.2 \mathrm{~V}$ (Fig. 6). A similar dependence was also observed in the LC cell with $180^{\circ}$ rotation of the director, studied in work [3]. To interpret the results, the depth of the ray penetration into the layer at different angles of incidence was calculated using the formula:

$$
z(\delta)=d-\frac{2 d}{\pi} \operatorname{asin} \sqrt{\frac{\left(1-\frac{n_{0}^{2}}{n_{g l}^{2} \sin \delta}\right) n_{e}^{2}}{n_{e}^{2}-n_{0}^{2}}},
$$




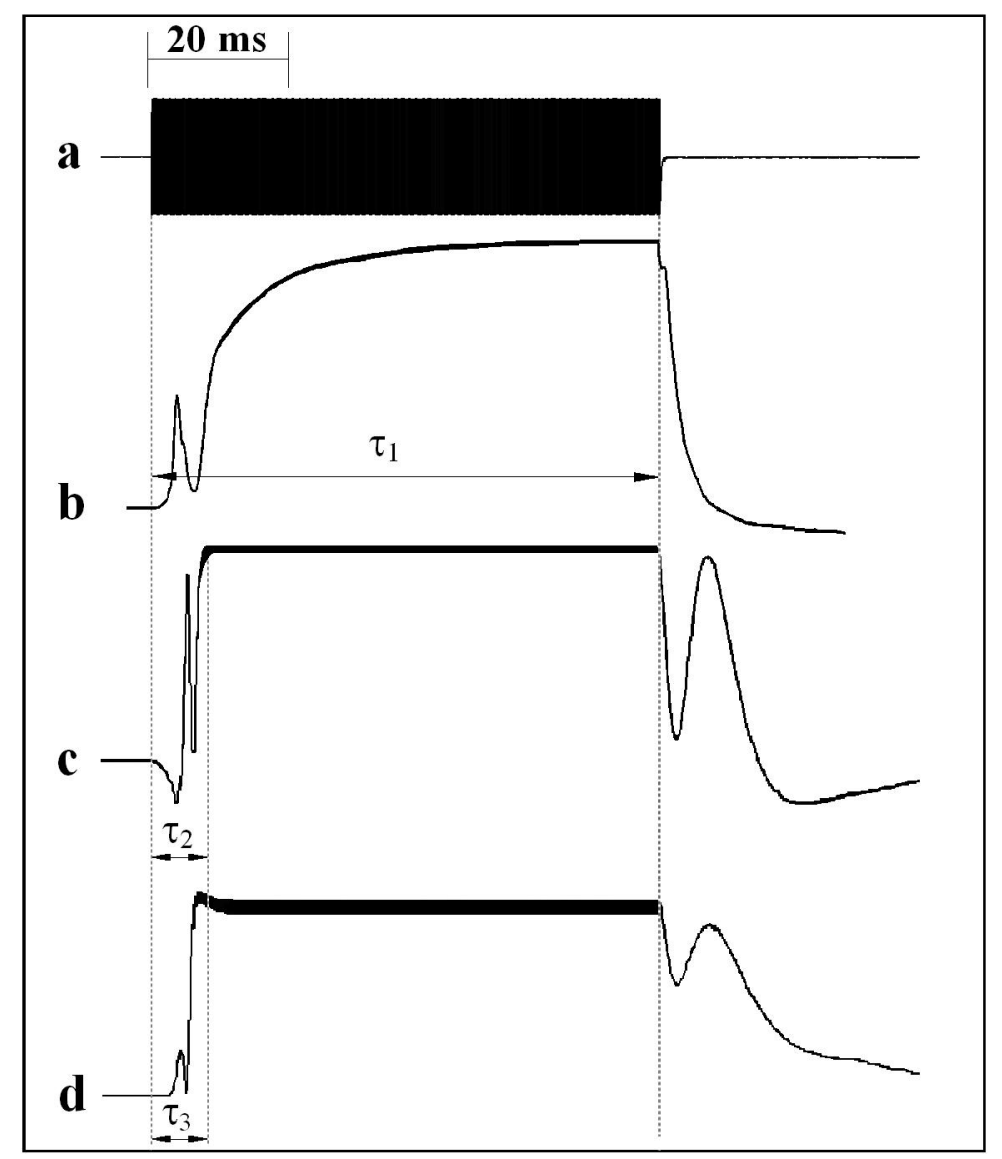

FIG. 4. Oscillograms of control voltage and optical response. (a) - Control voltage $U=8 \mathrm{~V}$, and carrier frequency $f=1000 \mathrm{~Hz}$. (b) - cut-off refraction mode, the angle of incidence of light on the layer $\delta=63^{\circ}, z=7.3 \mu \mathrm{m}$. (c) - electrically-controlled birefringence mode, (d) - cut-off Mauguin mode. Oscillograms (c) and (d) are obtained for the light incidence along the normal to the layer

where, $d$ - the thickness of the LC, $\delta$ - the angle of incidence for the extraordinary ray to the layer, $z$ - the depth of the ray penetration into the layer. The dependence of $z$ on $\delta$ is shown in Fig. 7. It is seen from Fig. 7 that as the angle of light incidence to the layer increases, the penetration depth decreases. When $z$ decreases, the dependence of $U_{\text {th }}$ on $z$ shows an increase in the threshold voltage. The increase of $U_{\text {th }}$ when $\delta$ increases, i.e. when the depth of the ray penetration into the layer decreases, can be attributed to the fact that the deviation of the LC director at the same angle close to the surface requires more voltage than in the layer depth [2]. It should be noted that a change of 1 in the angle of incidence results in a change of the ray penetration depth by $250 \mathrm{~nm}$ (Fig. 7). The latter provides a unique opportunity to study the local orientation structure of the LC director and its change dynamics in external electric fields. Therefore, we also studied the reorientation of the LC director upon switching off of the electric field for different angles of light incidence to the layer, and hence for the penetration depth $z$. The magnitude of the control voltage, disturbing the terms of refraction, exceeded the threshold voltage corresponding to all achievable values of $z$ and was the same $(U=8 \mathrm{~V})$ for all the angles of incidence (the depth of the ray penetration into the layer). In this case, in the layer with the equally deformed LC structure, we studied the relaxation of the local deformation of 


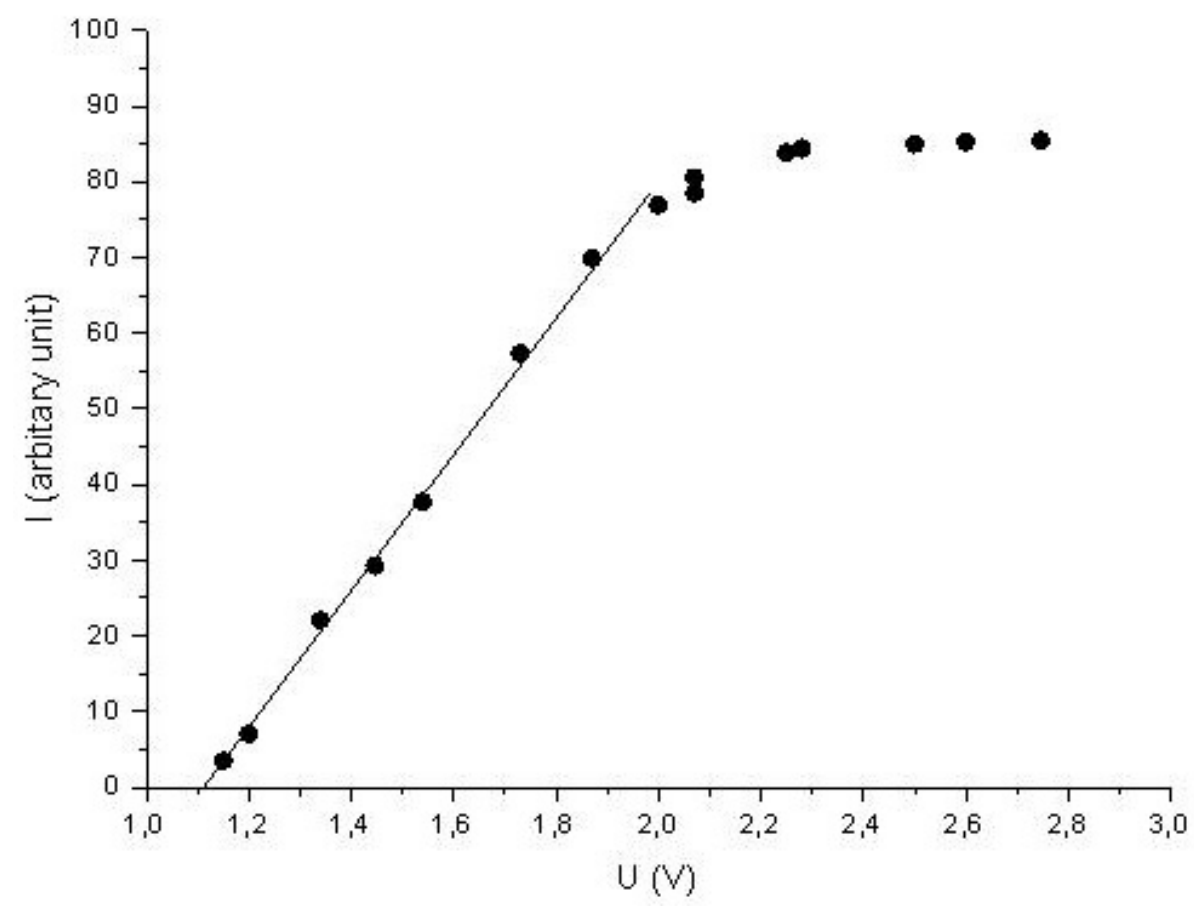

FIG. 5. The intensity of the transmitted light on the control voltage applied at an angle of incidence of the extraordinary ray in the LC layer. $\delta=63^{\circ}$

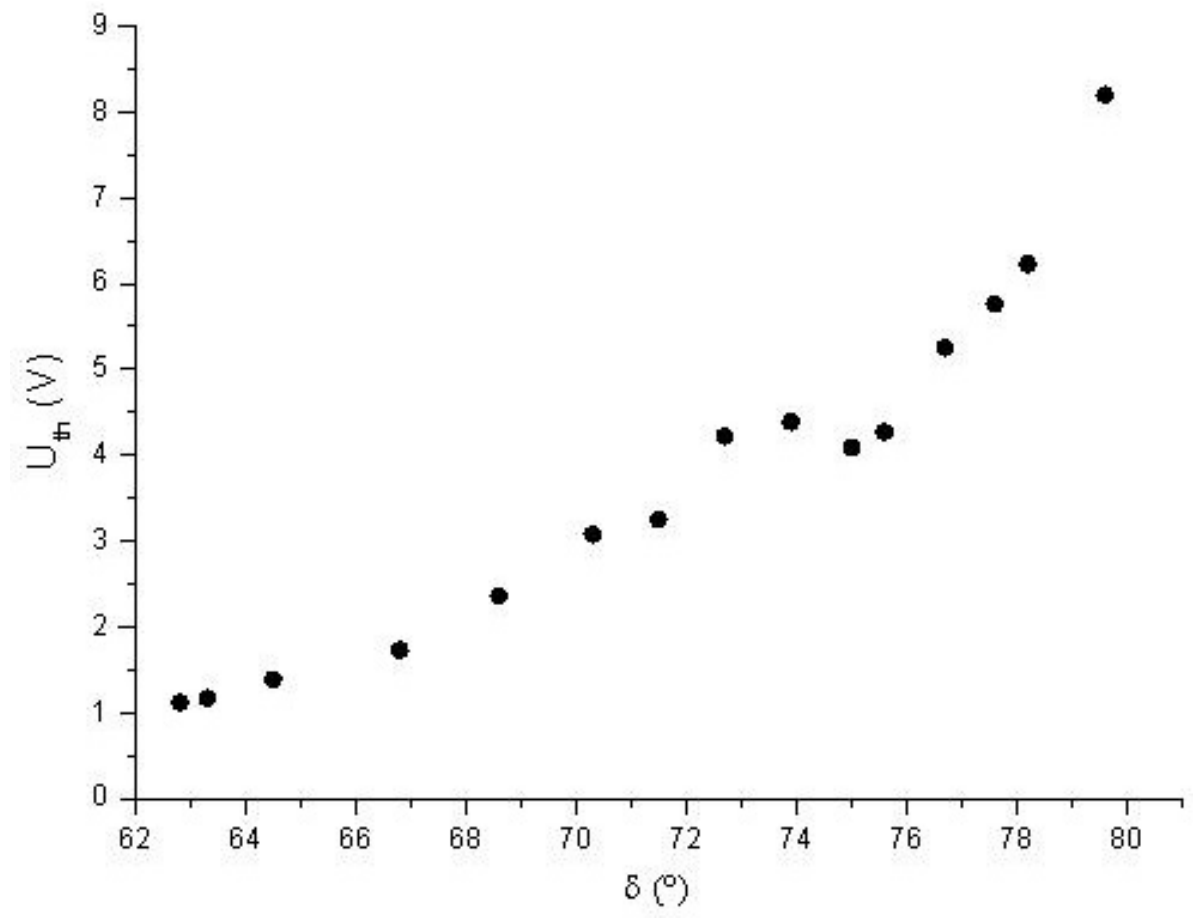

FIG. 6. The dependence of the threshold voltage $U_{\text {th }}$ on the angle of incidence of the extraordinary ray $\delta$ on the LC layer 
the director, which differed at different distances from the layer boundary. The recovery time of the optical transmission of the cell $\tau_{r}$ was determined using the oscillograms of the optical response to the electric field. The relaxation times correspond to the time taken to relax from $100 \%$ to $10 \%$ of the maximum transmission. As an example, the oscillograms for the optical response relaxation for several angles of incidence are shown in Fig. 8.

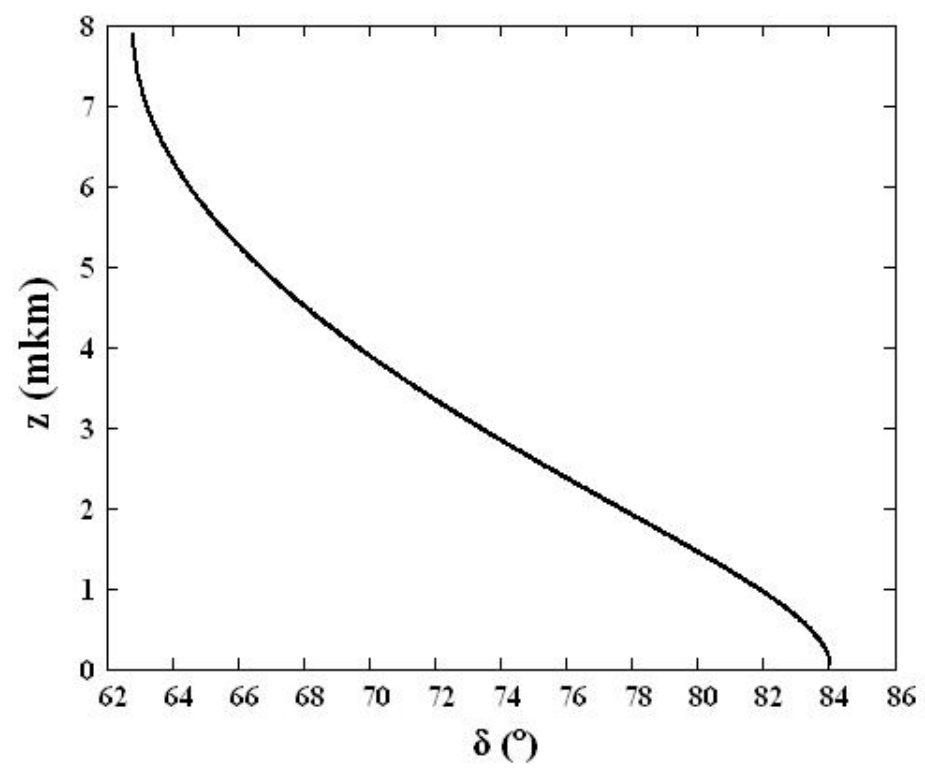

FIG. 7. The dependence of the penetration depth $z$ extraordinary ray in the liquid crystal layer on the incidence angle $\delta$

The dependence of $\tau_{r}$ on the depth of the ray penetration into the layer $z$ for the voltage of $8 \mathrm{~V}$ is shown in Fig. 9. The figure shows that the recovery time for optical transmission $\tau_{r}$ decreases along with the depth of the light penetration into the liquid crystal layer. Qualitatively, such dependence can be explained by the fact that the recovery rate of the LC initial orientation is proportional to the magnitude of elastic torque that affects the LC director. This elastic torque is at its maximum in the area of the LC layer, where there is a maximum orientation gradient of the director, when the field is off. We can assume that this is why $\tau_{r}$ decreases along with the distance from the boundary of the layer $z$ [5].

\section{Conclusion}

The refraction of light in the chiral liquid crystal layer (helical pitch of $32 \mu \mathrm{m}$ ) with the $90^{\circ}$ rotation of the director at different angles of incidence to the LC layer (the depth of the extraordinary ray penetration into the layer) has been studied. The effect of the electric field on the extraordinary ray refraction at different distances from the LC layer surface has been researched. The threshold character of the refraction cut-off (the light transmission of the cell) has been found. The dependencies of the threshold voltage of the refraction cut-off $U_{\text {th }}$ and the refraction recovery time $\tau_{r}$ of the electric field switching off on the depth of the ray penetration into the LC layer have been obtained.

The comparison of the results of the experimental research of the electric field influence on the extraordinary ray refraction in the helical liquid crystal structures with the $90^{\circ}$ and $180^{\circ}$ director rotation of the showed that in both structures, the minimum threshold voltage for the refraction disturbance $U_{t h(\min )}$ corresponds to the minimum angle of incidence to the layer. At 


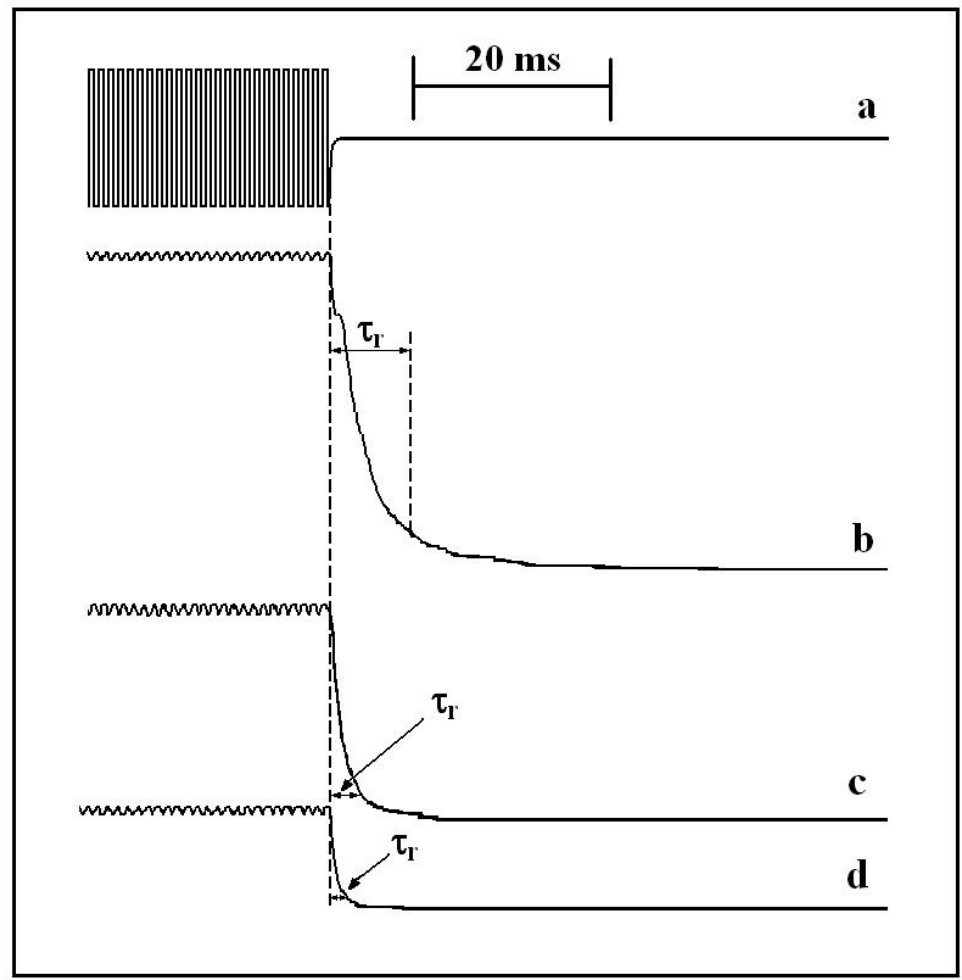

FIG. 8. The trailing edge of the optical response of the cell after the field at different depths of penetration of the beam in the LC layer. a) pulse voltage, $U=8 \mathrm{~V}, f=1 \mathrm{kHz} . \mathrm{b})-\mathrm{d})$ optical feedback for $z=7.2 \mu \mathrm{m}, z=5.1 \mu \mathrm{m}$ and $z=4.0 \mu \mathrm{m}$, respectively

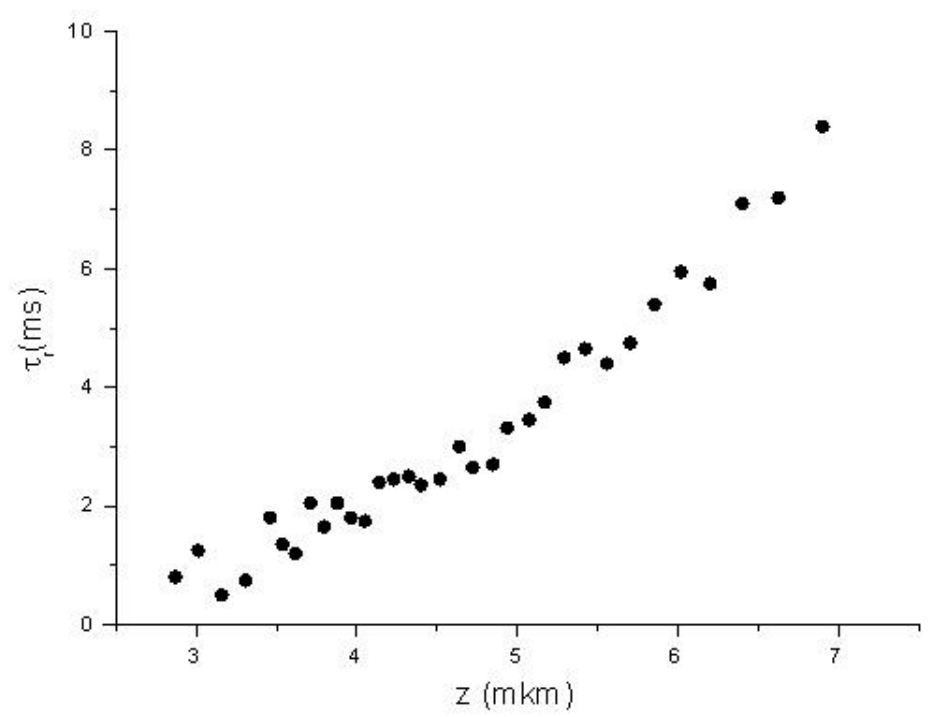

FIG. 9. Dependence of refraction recovery time $\tau$ on penetration depth $z$ extraordinary ray in the liquid crystal layer for voltage $8 \mathrm{~V}$ 
the same time, for the structure with the $180^{\circ}$ rotation of the director, $U_{\text {th }(\min )}$ causes refraction disturbance in the middle layer [3], and in the cell with $90^{\circ}$ rotation - at the layer boundary opposite to the input surface. The refraction recovery time $\tau_{r}$ is maximum in both structures for the minimum angle of incidence to the layer. For the structure with $180^{\circ}$ director rotation, $\tau_{r(\max )}$ corresponds to the director orientation recovery, necessary for recovering the refraction in the middle of the LC layer. At the same time, in the LC structure rotated $90^{\circ} \tau_{r(\max )}$ is required for the refraction recovery near the layer boundary opposite to the input surface, i.e. at the maximum depth of the ray penetration into the liquid crystal layer.

The study showed that the lowest threshold voltage for the refraction cut-off and the longest refraction recovery time corresponds to the maximum depth of the extraordinary ray penetration into the layer, regardless of the boundary conditions on the surfaces of the LC layer and the ray rotation point in the middle of the layer (rotation of the director by $180^{\circ}$ ), or at the far boundary of the layer (rotation of the director by $90^{\circ}$ ).

The theoretical background of the results is a rather complex issue, especially in the case of sufficiently strong electrical fields exceeding the Fréedericksz threshold. The solution to this problem is expected to be found at some point in the future.

Thus, the combined experimental and theoretical research of the light refraction in helical-structured liquid crystals and the effect of electric field on refraction will allow investigation of the process for local director reorientation at different distances from the interfaces between liquid crystals and glass.

\section{References}

[1] E.V. Aksenova, A.A. Karetnikov, A.P. Kovshik et al. Return back of the extraordinary beam for oblique incidence in helical liquid crystals with large pitch. Europhys. Lett., 2005, 69, P. 68-74.

[2] E.V. Aksenova, A.A. Karetnikov, A.P. Kovshik et al. Propagation of Light through a Forbidden Zone in Chiral Media. J. Opt. So. Amer., 2008, 6 (104), P. 909-919.

[3] A.A. Karetnikov, N.A. Karetnikov, A.P. Kovshik, et al. Electric Field Effect on Refraction of Light in a Layer of Chiral Liquid Crystal with Large Pitch. Opt. Spectr., 2010, 6 (108), P. 947-953.

[4] A.A. Karetnikov, N.A. Karetnikov, A.P. Kovshik et al. Local Dynamics of Director Reorientation under Electric Field in Helical LC Structure. Mol. Cryst. Liq. Cryst., 2012, 561, P. 97-106.

[5] E.V. Aksenova, B.B. Divinsky, A.A. Karetnikov, et al. Particular the propagation of light in the cells of chiral liquid crystals in an external electric field. Journal of Experimental and Theoretical Physics, 2014, 2 (145), P. 369-380. 